\title{
Can Vemurafenib Induce Vitiligo in Metastatic Melanoma Patients?
}

\author{
Ricardo Ruiz-Villaverde ${ }^{1}$, Daniel Sánchez-Cano ${ }^{2}$ \\ ${ }^{1}$ Dermatology Unit, Hospital Universitario Virgen de las Nieves, Granada, Spain \\ ${ }^{2}$ Internal Medicine Unit, Santa Ana Hospital, Motril, Granada, Spain
}

To the Editor,

Melanoma is a mucocutaneous or ocular, sporadic or familial, neoplasm that is associated with a range of genetic factors. Environmental predisposing factors include the exposure to intense sunlight associated with sunburn, as well as a type II phototype. Between $1.5 \%$ and $20 \%$ of patients with melanoma may have vitiligo-like lesions. From a clinical point of view, the latter may be classified as: a) lesions adjacent to the primary melanoma or metastatic regression; b) development of a melanocytic nevi nevus halo on pre-existing nevi; or c) vitiliginous lesions at sites distant to the melanoma, whether isolated or associated with the ocular findings of Vogt-KoyanagiHarada syndrome. We report this relationship of vitiligo-like lesions in a patient with a metastatic melanoma who attended our Unit for treatment with vemurafenib.

A 56 year-old male, with no past medical history, was referred to our outpatient clinic complaining about a cutaneous lesion on his back, which had developed abnormal changes for 2 years. With a clinical diagnosis of typical nevi, the patient had the lesion excised. Histological examination revealed superficial spreading melanoma (SSM), absence of ulceration, a $1.9 \mathrm{~mm}$ Breslow Depth Index, and a Clark IV Level with positive sentinel node.

An elective lymph node dissection was required, and the presence of micrometastases was observed in three nodes (Stage IIIA). BRAF (Serine Threonine kinase B raf) V600E mutation in primary tumor and lymph node were analyzed, with a positive result in both cases. After evaluation of the case by our Hospital Oncological Committee, treatment with 960 mg Vemurafenib (Roche; Basel, Switzerland) twice daily was started.

Eight weeks later, vitiligo-like depigmented macules developed on the patient's neckline (Figure 1). The examination with a Wood's lamp revealed similar lesions on the periocular and perioral area. A skin biopsy was then performed, showing a loss of melanocytes on the basal layer in the excised area.

After ten months of treatment, the patient remains tumorfree, according to the imaging tests performed, and is under periodic follow-ups by the Units of Dermatology and Oncology. Ethics committee approved the presentation of this case report with academic and research purpose under signed of the informed consent by our patient.

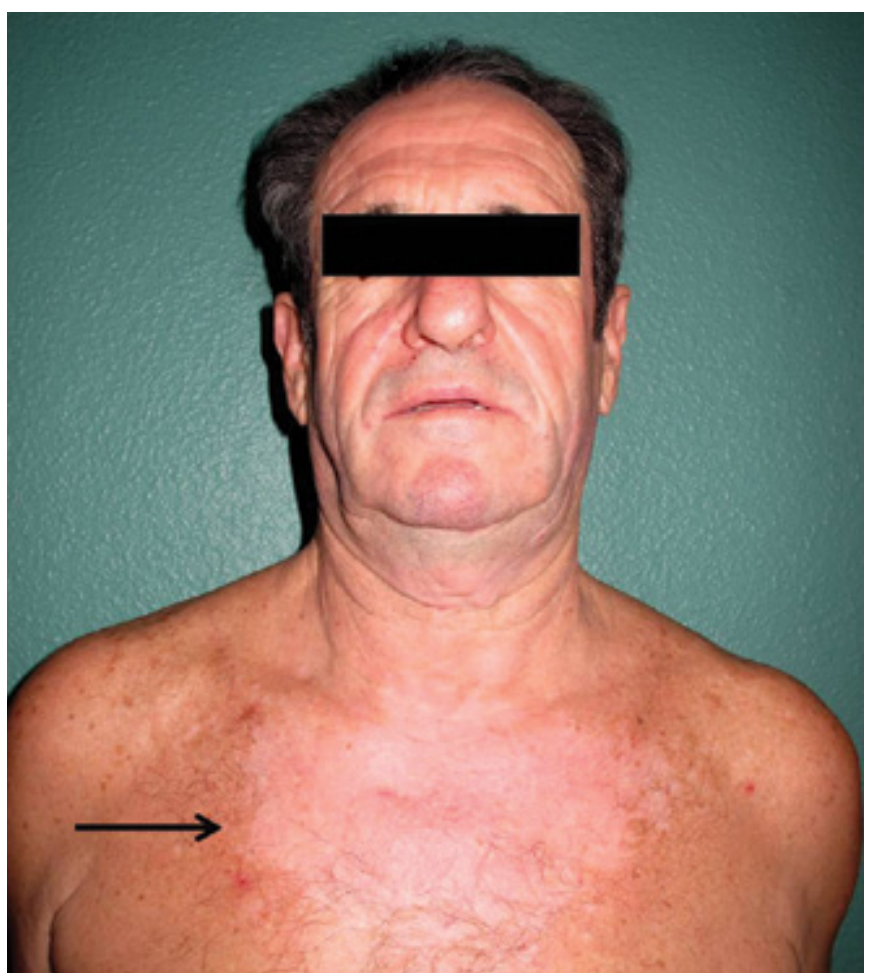

FIG. 1. Vitiligo-like lesion on the neckline of our patient 
Vitiligo-like lesions occur most commonly in the setting of untreated metastatic melanoma, although they also develop in association with immunomodulatory therapies, such as INFa2b, IL2A, and the new biologics drugs (BRAF inhibitors, anti-CTLA-4 antibody therapy and anti MEK-MAPkinse ERK kinase-drugs). In these cases, they are usually considered grade 1 toxicity, i.e. a mild adverse effect, and therefore, no further action is required.

Drug-induced vitiligo shows different characteristics to classical vitiligo from the epidemiological point of view: an absent family history, partial discoloration, a wayward patchy distribution and a rapid development after the introduction of the drug, as in our case (1).

The etiology of the occurrence of this phenomenon remains unclear, although the main theory considers it the result of metabolic dysregulation and damage by direct cytotoxicity against melanocytes as well (2). On the other hand, the use of these drugs that selectively alter the immune system may explain the appearance of vitiligo as an immune-related adverse event.

The development of vitiligo-like lesions in metastatic melanoma under treatment with interferon has also been described (3), and more recently, with the BRAF inhibitor Vemurafenib, adding further complexity to the association of both diseases (4). However, what it does seem clear is that the development of vitiligo-like lesions in both untreated patients and patients on biological therapy or interferon- $\alpha 2 \beta$ improves the survival rates for metastatic melanoma (5).

Ethics Committee Approval: Ethics committee approval was received for this study from the ethics committee of the university.

Informed Consent: Written informed consent was obtained from patient who participated in this study.

Peer-review: Externally peer-reviewed.
Author contributions: Concept - R.R.V., D.S.C.; Design - R.R.V.; Supervision - R.R.V., D.S.C.; Resource - R.R.V.; Materials - R.R.V., D.S.C.; Data Collection \&/or Processing - R.R.V., D.S.C.; Analysis \&/or Interpretation - R.R.V.; Literature Search - R.V.; Writing D.S.C.; Critical Reviews - D.S.C., R.R.V.

Conflict of Interest: No conflict of interest was declared by the authors.

Financial Disclosure: The authors declared that this study has received no financial support.

\section{REFERENCES}

1. Hartmann A, Bedenk C, Keikavoussi P, Becker JC, Hamm H, Bröcker EB. Vitiligo and melanoma-associated hypopigmentation (MAH): shared and discriminative features. J Dtsch Dermatol Ges 2008;6:1053-9. [CrossRef]

2. Ram M, Shoenfeld Y. Harnessing autoimmunity (vitiligo) to treat melanoma: A myth or reality? Ann NY Acad Sci 2007;1110:41025. [CrossRef]

3. García-F-Vilalta MJ, Ruiz-Genao D, Ríos L, Fraga J, García-Diez A, Fernández-Herrera J. Leucodermia en pacientes con melanoma tratados con interferón. Actas Dermosifiliogr 2003;94:173-5. [CrossRef]

4. Alonso-Castro L, Ríos-Buceta L, Vano-Galvan S, Moreno C, Soria-Rivas A, Jaén P. Vitiligo in 2 patients receiving vemurafenib for metastatic melanoma. J Am Acad Dermatol 2013;69:e28-9. [CrossRef]

5. Quaglino P, Marenco F, Osella-Abate S, Cappello N, Ortoncelli $\mathrm{M}$, Salomone B, et al. Vitiligo is an independent favourable prognostic factor in stage III and IV metastatic melanoma patients: results from a single-institution hospital-based observational cohort study. Ann Oncol 2010;21:409-14. [CrossRef] 